\title{
Serotonin: beyond menopause
}

\author{
Mara Carsote ${ }^{1,2}$, Eugenia Nedeltcheva Petrova ${ }^{2}$, Otilia Radu ${ }^{1}$, Andrei Goldstein $^{2}$, Adina Ghemigian ${ }^{1,2}$, \\ Ana Valea ${ }^{3}$ \\ 1"Carol Davila" University of Medicine and Pharmacy, Bucharest \\ 2"C.I. Parhon" National Institute of Endocrinology, Bucharest \\ 3 "Iuliu Hatieganu" University of Medicine and Pharmacy, Clinical County Hospital, Cluj-Napoca
}

\begin{abstract}
Serotonin is a brain neurotransmitter, a local gut-produced pro-kinetic agent and a platelets-released monoamine with haemostasis role. We focus on revealing the serotonin interferences with menopause mainly related to climacteric syndrome and bone health. This is a short commentary type of manuscript, centred on English language PubMed research. Serotonin is connected to the hypothalamic thresholds for temperature regulation in vasomotor symptoms that is why drugs that elevate the serotonin synaptic plaque exposure like SSRI (serotonin selective reuptake inhibitors) are indicated. Serotonin is also linked to bone field but its testing into the blood has not been unanimously found to be correlated with bone mineral density or menopausal bone remodelling markers. Conditions with age-related onset as carcinoid tumours bring a serotonin excess but not necessary a bone loss. From climacteric syndrome to bone regulation and to pathological domain of neuroendocrine neoplasia, serotonin plays a complex role on menopause's field.
\end{abstract}

Keywords: serotonin, bone, climacteric syndrome

\section{INTRODUCTION}

Serotonin, also named 5-hydroxytryptamine, is a molecule serving as central neurotransmitter, as local gut-produced pro-kinetic agent and a platelets-released monoamine with haemostasis role (1-3). A part from all these, serotonin is connected to the hypothalamic thresholds for temperature regulation in climacteric vasomotor symptoms that is why drugs that elevate the serotonin synaptic plaque exposure like SSRI (serotonin selective reuptake inhibitors) are indicated for menopausal complains (4). Serotonin is also linked to bone field by central positive actions that antagonises peripheral negative effects of intestine-derived 5-hydroxytryptamine (5).

\section{OBJECTIVE}

We focus on revealing the serotonin interferences with menopause mainly related to climacteric syndrome and bone health.

\section{MATERIAL AND METHOD}

This is a short commentary type of manuscript. The research is centred on English language written articles recently published in journals indexed in PubMed database.

\section{RESULTS}

Climacteric syndrome includes hot flashes and mood disturbances described in up to $70 \%$ of women and one third of females have these complains starting from peri-menopause (6-10). Traditionally, vasomotor symptoms are hot flashes and night sweats; a wave may last from one to five minutes (6-10). These aspects are presented up to 5 but even to 10 years depending on each person (mostly one to four years) (6-10). The flashes do not necessarily have a well revealed trigger as caffeine, physical and emotional stress (6-10). The underling mechanisms are represented by decreased levels of 
Serotonin:

aspects interfering with menopausal status

Indirect bone formation

Vasomotor symptoms

\begin{tabular}{|c|}
\hline Direct bone loss \\
\hline Haemostasis \\
\hline Intestinal motility \\
\hline
\end{tabular}

Physiology

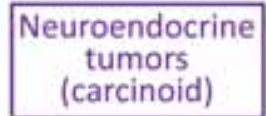

Pathology
FIGURE 1. Synthesis of main actions of serotonin that interfere with normal and pathological status, especially in menopause

estrogens \& progesterone that trigger neuroendocrine systems as serotonin and norepinephrine which cause a thermoregulatory dysfunction in hypothalamus reflected by higher core body temperatures as well as narrowing of thermoregulatory zone (6-10). The practical application of serotonin involvement is represented by the use of SSRIs for treatment of climacteric- related vasomotor disturbances especially in circumstances where estrogens are contra-indicated as incidental breast cancer or where concurrent depression symptoms may be reduced by antidepressants with dual function (6-10).

Regarding the bone, circulating serotonin has been found to be correlated with femoral areal bone mineral density in menopausal women but not in men (5). The OSTPRE study found that anti-depressive drugs which target serotoninergic pathways cause menopausal decrease of bone mineral density, a process that is accelerated by a higher drug dose (11). Is seems that an additional risk for osteoporotic fractures is brought by risk of falling caused by depression and associated therapy as well as co-morbidities with a higher risk on menopause as visual and blood pressure disturbances or neurological conditions (12). However, SSRI- related bone loss is also seen in premenopausal and younger women $(13,14)$. In menopause, circulating serotonin, even involved in bone turnover, does not accurately predict future bone loss, potentially due to estrogen- independent mechanisms (15).

\section{DISCUSSION}

Regarding prior published manuscripts from Romanian experience, we mention the large use of blood serotonin assays as neuroendocrine marker for carcinoid tumours which became easily accessible during the last years since the therapy with somatostatin analogues is country wide available (16-18). This aspect includes menopausal population knowing that the onset of neuroendocrine neoplasia is age-related with a much higher chance to affect women in menopause rather than those in pre-menopause (16-18). Whether osteoporosis in these subjects is strictly menopausal due to estrogens lack or elevated serotonin as effect of tumour production represents a contributor to bone loss is still an open question (19). Another experience is related to circulating 5-hydroxytryptamine assessment targeting the possibility of becoming a bone remodelling marker (20). A study on 191 menopausal females having an average age of 57.1 years who were anti-osteoporotic drugs naïve showed a weak correlation between serotonin and bone formation marker osteocalcin in patients with osteoporosis and between the monoamine and bone formation marker alkaline phosphatase in subjects with osteopenia but not with DXA parameters (20). These results pointed that serotonin is still difficult to be framed in particular menopauselinked skeleton context.

\section{CONCLUSION}

From vasomotors symptoms included in climacteric syndrome to bone regulation and to pathological domain of carcinoid tumours, serotonin plays a complex role on menopause's field.

Acknowledgement: none

Conflict of interest: The research is part of Carol Davila UMPh project 33878/11.11.2014/ Young researchers 


\section{REFERENCES}

1. Homberg J.R., Schubert D., Asan E., Aron E.N. Sensory processing sensitivity and serotonin gene variance: Insights into mechanisms shaping environmental sensitivity. Neurosci Biobehav Rev. 2016 Sep 30;71:472-483.

2. Kendig D.M., Grider J.R. Serotonin and colonic motility. Neurogastroenterol Motil. $2015 \mathrm{Jul} ; 27(7): 899-905$.

3. Warkentin T.E., Arnold D.M., Nazi I., Kelton J.G. The platelet serotonin-release assay. Am J Hematol. 2015 Jun;90(6):56472.

4. Palacios S., Mejias A. An update on drugs for the treatment of menopausal symptoms. Expert Opin Pharmacother. 2015;16(16):2437-47.

5. Wang Q., Chen D., Nicholson P. et al. The associations of serum serotonin with bone traits are age- and gender-specific. PLoS One. 2014 Oct 3;9(10):e109028. doi: 10.1371/journal.pone.0109028. eCollection 2014.

6. Bezerra A.G., Andersen M.L., Tufik S., Hachul H. Approach towards mild depression: shortest way to treat climacteric syndrome? Maturitas. 2013 Jan;74(1):105.

7. Albertazzi P. Noradrenergic and serotonergic modulation to treat vasomotor symptoms. J Br Menopause Soc. 2006 Mar;12(1):7-11.
8. Imai A., Matsunami K., Takagi H., Ichigo S. New generation nonhormonal management for hot flashes. Gynecol Endocrinol. 2013 Jan;29(1):63-6.

9. Stuenkel C.A., Davis S.R., Gompel A., et al. Treatment of Symptoms of the Menopause: An Endocrine Society Clinical Practice Guideline. J Clin Endocrinol Metab. 2015 Nov; 100(11):3975-4011.

10. Neves-E-Castro M., Birkhauser M., Samsioe G. et al. EMAS position statement: The ten point guide to the integral management of menopausal health. Maturitas. 2015 May;81(1):88-92.

11. Rauma P.H., Honkanen R.J., Williams L.J. et al. Effects of antidepressants on postmenopausal bone loss - A 5-year longitudinal study from the OSTPRE cohort. Bone. 2016 Aug;89:25-31.

12. Warden S.J., Fuchs R.K. Do Selective Serotonin Reuptake Inhibitors (SSRIs) Cause Fractures? Curr Osteoporos Rep. 2016 Oct;14(5):211-8.

13. Sheu Y.H., Lanteigne A., Stürmer T. et al. SSRI use and risk of fractures among perimenopausal women without mental disorders. Inj Prev. 2015 Dec;21(6):397403.

14. Moura C., Bernatsky S., Abrahamowicz M. et al. Antidepressant use and 10-year incident fracture risk: the population-based
Canadian Multicentre Osteoporosis Study (CaMoS). Osteoporos Int. 2014 May; 25(5):1473-81.

15. Kim H.Y., Park M.H., Yoon H.K., Han K.O. The changes in plasma serotonin levels after hormone therapy and their relationship with estrogen responsiveness on bone in postmenopausal women. J Clin Endocrinol Metab. 2012 Jun;97(6):1986-94.

16. Carsote M., Paun S., Neamtu M.C. et al. The immunohistochemistry aspects in two cases of neurofibromatosis-associated abdominal tumors. Rom J Morphol Embryol. 2012:53(2):401-5.

17. Poiana C., Neamtu M.C., Avramescu E.T. et al. The dedifferentiation of neuroendocrine tumor metastases: myth or reality? Rom J Morphol Embryol. 2013; 54(1):201-3.

18. Poiana C., Carsote M., Ardeleanu C. et al. The value of the immunohistochemistry in a case of gastric neuroendocrine tumor and thyroid metastasis. Rom J Morphol Embryol. 2011;52(1):187-92.

19. Poiana C., Carsote M., Neamtu M.C., et al. Well-differentiated neuroendocrine tumor and osteoporosis: incidental findings? Rom J Morphol Embryol. 2013;54(4):1169-71.

20. Carsote M., Radoi V., Geleriu A. et al. The serotonin and the bone assessment. J Med Life. 2013 Jun 15;6(2):151-5. 